\title{
Factores sociodemográficos y laborales relacionados con la exposición laboral a riesgos psicosociales por ocupación en España. Proyecto MatEmESp
}

\author{
M. Carmen González-Galarzo ${ }^{a, b}$, Elena Ronda ${ }^{b, c, d}$, \\ Fernando G. Benavides ${ }^{b, d, e}$ y Ana M. Garcia ${ }^{d, f}$
}

DOI: 10.12961/aprl.2019.22.03.2

Recibido: 18 de marzo de 2019

Aceptado: 23 de mayo de 2019

\section{RESUMEN}

Objetivo: El objetivo del estudio es describir el nivel de exposición a riesgos psicosociales por ocupación en población laboral española y analizar su relación con condiciones de empleo y características sociodemográficas.

Métodos: La Matriz Empleo Exposición Española (MatEmESp) es la fuente de información para la descripción de los niveles de exposición a riesgos psicosociales en 2005. Se identifican las ocupaciones con mayores niveles de exposición y se analiza su relación con condiciones de empleo y características sociodemográficas por ocupación.

Resultados: Se encontraron niveles de exposición a influencia en el trabajo inferiores al punto medio de la escala (50, escala de 0 a 100) y a ritmo en el trabajo ligeramente por encima $(\bar{x}=46,8$ y $\bar{x}=50,8)$. Los niveles más elevados de exposición estaban en ocupaciones manuales, algunas cualificadas presentaban también alta inseguridad (Economistas) y ritmo de trabajo (Directores/as). Conforme disminuía el nivel de clase social y de estudios, aumentaba la inseguridad ( $r h o=0,45$ y 0,38 respectivamente), y disminuía el apoyo de los compañeros $(\mathrm{rho}=-0,46$ y $-0,48)$ y la influencia en el trabajo ( $r h o=-0,73$ y -0,63). Conforme aumentaba la proporción de extranjeros, disminuía el apoyo e influencia (rho=-0,44 y -0,43); conforme aumentaba el número de asalariados, disminuía la influencia (rho=-0,38); el aumento en contratos temporales, disminuía el apoyo de los compañeros $(\mathrm{rho}=-0,34)$ e influencia $(\mathrm{rho}=-0,53)$; y conforme aumentaba el número de horas extraordinarias, aumentaba la influencia $(\mathrm{rho}=0,49)$.

Conclusiones: Este estudio confirma la existencia de desigualdades en la exposición a riesgos psicosociales en el trabajo en función de la ocupación y las características sociodemográficas y condiciones de empleo de la ocupación desempeñada.

PALABRAS CLAVE: Estrés laboral, exposición laboral, factores socioeconómicos, empleo, clasificación, ocupaciones.

\section{SOCIODEMOGRAPHIC AND OCCUPATIONAL FACTORS RELATED TO EXPOSURE TO PSYCHOSOCIAL RISK FACTORS BY OCCUPATION IN SPAIN. THE MATEMESP PROJECT}

\section{ABSTRACT}

Objective: The objective of the study is to describe the level of exposure to psychosocial risk factors by occupation in the Spanish working population and analyze their relationship with sociodemographic characteristics and employment conditions.

Methods: The Spanish Job-Exposure Matrix (MatEmESp) is used to describe the levels of exposure to psychosocial risk factors in 2005. We identified occupations with higher levels of exposure and analysed their relationship with employment conditions and sociodemographic characteristics.

Results: Levels of exposure to work influence were below the midpoint (50, on a scale from 0 to 100$)$; for work pace, they were slightly above $(\bar{x}=46.8$ and $\bar{x}=50.8$, respectively). The highest levels of exposure were found in manual occupations; some skilled occupations were also associated with high insecurity (economists) and work pace (managers). As social class and education decreased, insecurity increased $(\mathrm{rho}=0.45$ and 0.38 respectively), and both co-worker support ( $\mathrm{rho}=-0.46$ y -0.48 , respectively) and influence (rho $=-0.73$ y -0.63 , respec-

a. Universidad de Valencia. Departamento de Psicología Evolutiva y de la Educación, Valencia, España.

b. Centro de Investigación en Salud Laboral. Universidad Pompeu Fabra, Barcelona, España.

c. Universidad de Alicante. Departamento de Enfermería comunitaria, Medicina Preventiva, Salud Pública e Historia de la Ciencia. Alicante, España. d. CIBER Epidemiología y Salud pública (CIBERESP), Madrid, España. e. Instituto Hospital del Mar de Investigaciones Médicas, Barcelona, España. f. Universidad de Valencia. Departamento de Medicina Preventiva y Salud Pública, Ciencias de la Alimentación, Toxicología y Medicina Legal. Valencia, España.
Autora para la correspondencia:

M. Carmen González Galarzo

Departamento de Psicología Evolutiva y de la Educación. Facultad de Psicología. Universidad de Valencia

Avda. Blasco Ibáñez, no 21. 46010. Valencia

Telf: 963983553

Correo electrónico: maria.c.gonzalez-galarzo@uv.es

Financiación: Instituto de Salud Carlos III (referencia PI081496),

Conselleria de Sanitat de la Generalitat Valenciana (referencia .066/2009),

Instituto Nacional de Seguridad e Higiene en el Trabajo

and Fundación Prevent. 
tively) decreased. As the proportion of foreigners increased, support and influence decreased (rho $=-0.44$ and -0.43 , respectively). As the number of employees increased, work influence decreased ( $\mathrm{rho}=-0.38$ ). I Increases in temporary contracts were associated with a decrease in co-worker support ( $\mathrm{rho}=-0.34)$ and influence $(\mathrm{rho}=-0.53)$ and, as overtime increased, work influence increased $(\mathrm{rho}=0.49)$.

Conclusion: This study confirms the presence of inequalities in exposure to psychosocial risk factors at work, depending on the occupation and the sociodemographic characteristics and employment conditions of the occupation performed.

KEYWORDS: occupational stress, occupational exposure, socioeconomic factors, employment, classification, occupations.

\section{INTRODUCCIÓN}

Una matriz empleo-exposición (MEE) es una base de datos que detalla exposiciones laborales y otras características de los trabajadores presentes en cada ocupación ${ }^{1}$. En España se han llevado a cabo estudios que han utilizado información de $\mathrm{MEE}^{2}$, pero hasta 2012, fecha en que fue accesible online MatEmESp ${ }^{3}$, no se dispuso de una MEE general para población laboral española. MatEmESp contiene información sobre la exposición a agentes de higiene, de seguridad, ergonómicos y psicosociales, y sobre las condiciones de empleo y características sociodemográficas de los trabajadores por ocupación. Se puede obtener el detalle de la metodología de MatEmESp en una publicación previa ${ }^{4}$. Otros países europeos también han desarrollado matrices generales, como Finlandia ${ }^{1}$, Dinamarca ${ }^{5}$, Francia ${ }^{6}$ o Suecia .

La información contenida en MatEmESp se ha usado previamente para describir la exposición a riesgos ergonómicos y los factores asociados ${ }^{8}$. El estudio multicaso-control poblacional (MCC-SPAIN) que investiga la influencia de factores ambientales y su interacción con factores genéticos en tumores en España, también ha utilizado esta fuente de datos. El proyecto DDM-Occup, que forma parte de este estudio, ha publicado un análisis de la relación entre la exposición laboral a agentes higiénicos con la densidad mamaria9. Otras publicaciones relacionadas con MatEmESp se detallan en su página web ${ }^{10}$.

En los últimos años, la crisis económica ha llevado a un deterioro de las condiciones laborales, también en el contexto psicosocial ${ }^{11,12}$. La exposición a riesgos psicosociales se ha relacionado con enfermedades cardiovasculares ${ }^{13}$, salud mental ${ }^{14}$ trastornos musculoesqueléticos ${ }^{15}$ y el consumo de alcohol y tabaco ${ }^{16}$.

Describir las ocupaciones con mayores niveles de exposición a riesgos psicosociales, así como identificar los colectivos más desfavorecidos, puede ayudar en la planificación de los programas preventivos y a identificar enfermedades profesionales relacionadas con esta exposición. El objetivo de este trabajo es describir el nivel de exposición a los principales riesgos psicosociales por ocupación en población laboral española, y su relación con factores sociodemográficos y condiciones de empleo, según la información contenida en MatEmESp.

\section{MATERIAL Y MÉTODOS}

MatEmESp incluye estimaciones de la exposición a los siguientes factores psicosociales: apoyo social de los compañeros, apoyo social del supervisor, estima, exigencias emocionales, exigencias psicológicas cuantitativas, influencia, inseguridad y empleabilidad, posibilidad de desarrollo y ritmo de trabajo. El estimador disponible es intensidad de la exposición (media, mediana y desviación típica) en cada ocupación, en una escala estandarizada de 0-100, en base a las puntuaciones de cada factor, basadas en uno o varios ítems. Por las características de su construcción ${ }^{4}$, MatEmESp incluye sólo información sobre la exposición a estos factores psicosociales en 84 ocupaciones a tres dígitos de la $\mathrm{CNO}$ 94. Toda la información sobre las características de MatEmESp, así como sus contenidos, se puede consultar online ${ }^{10}$.

MatEmESp también contiene información sobre las condiciones de empleo y características sociodemográficas de los trabajadores por ocupación. En este trabajo se describe el nivel de exposición a factores psicosociales por ocupación en población laboral española en el año 2005, en base a la información contenida en MatEmESp. Se seleccionaron los cuatro agentes que consideramos más representativos de los principales modelos explicativos del estrés laboral, Apoyo-demanda-control (Apoyo de los compañeros, ritmo de trabajo e influencia en el trabajo) y Esfuerzo-Recompensa (Inseguridad), para identificar, posteriormente, las cinco ocupaciones con mayor riesgo psicosocial a cada uno de estos factores. Se analiza también la relación entre el nivel de exposición a estos agentes y las características sociodemográficas: edad (menos de 31 años, 31-45 años y más de 45 años), sexo, nivel de estudios (sin estudios, primarios, secundarios, universitarios), país de origen (España, otros); y laborales en cada ocupación, Clase Social ocupacional (categorías I-V) ${ }^{17}$, situación profesional (empleador, autónomo, asalariado o ayuda familiar), tipo de contrato (temporal), tipo de jornada (tiempo parcial), horas extraordinarias, y la media de horas de trabajo semanales. Para explorar la relación entre los factores psicosociales y las características de las ocupaciones, se realizan gráficos de dispersión con curvas suavizadas obtenidas mediante el método Loess y se calculan los coeficientes de correlación de Spearman (rho) para analizar estas relaciones. Se han considerado correlaciones altas cuando el coeficiente era mayor de 0,70 y moderadas entre $0,30 \mathrm{y}$ 0,70 , ambos inclusive. Los datos se analizan con el paquete estadístico SPSS versión ${ }^{18}$.

\section{RESULTADOS}

Las puntuaciones de exposición de los agentes psicosociales analizados, pueden clasificarse en función del sentido de la interpretación del valor de la exposición. Por ejemplo, encontramos agentes en los que una mayor puntuación indica que hay mayor riesgo psicosocial (mayor puntuación, situación más desfavorable), es el caso de Ritmo de trabajo; y otros en los que una menor 
puntuación indica que hay un mayor riesgo psicosocial (menor puntuación, situación más desfavorable), el caso de Influencia en el trabajo. Considerando el conjunto de ocupaciones con información disponible en MatEmESp, y la forma de interpretación de los resultados, se puede afirmar que, en general, las estimaciones de exposición indican que no hay un elevado riesgo psicosocial en los trabajadores españoles en el año analizado. De los datos desagregados por factor de riesgo psicosocial, se destaca que el apoyo social de los compañeros y de los supervisores y el nivel de estima es valorado de forma positiva en la población de referencia, mostrándose puntuaciones por encima del punto medio de la escala ${ }^{50}$. Sin embargo, tanto el nivel de influencia en el trabajo, como el ritmo de trabajo es valorado de forma negativa (tabla I).

En la tabla II se muestran las cinco ocupaciones con mayor nivel de exposición (puntuaciones más alejadas, en sentido de mayor riesgo psicosocial, de la media poblacional) a cada uno de los factores psicosociales. En lo que se refiere al nivel de apoyo social de los compañeros declarado, la ocupación de "Empleadas de hogar" es la única que declaró un bajo nivel de apoyo (por debajo del punto medio de la escala, 50). Sin embargo, también encontramos otras ocupaciones con valores de este tipo de apoyo por debajo de la media de referencia (65), ocupaciones relacionadas con la limpieza, cuidado de personas y conductores. Los menores niveles de influencia en el trabajo también se encuentran en ocupaciones poco cualificadas, relacionados con la limpieza, ordenanzas y peones de la construcción e industria, todas ellas con puntuaciones inferiores a la media $(46,8)$. Sin embargo, niveles altos de inseguridad (superiores a la media: 42) se encontraban en ocupaciones cualificadas, como profesionales del derecho, o de la administración de empresas (Economistas) y menos cualificadas de la industria y construcción. Igualmente, encontramos un ritmo elevado de trabajo, más de 10 puntos por encima de la media poblacional $(50,8)$, tanto en ocupaciones cualificadas relacionadas con la dirección de empresas, como en trabajadores menos cualificados del sector de la restauración.

En la tabla III se encuentran el análisis de la relación entre el nivel de exposición a riesgos psicosociales y las características sociodemográficas y las condiciones de empleo por ocupación. No se ha encontrado una relación significativa entre el nivel de exposición a los factores psicosociales y la proporción de trabajadores según sexo en cada ocupación. Las ocupaciones menos cualificadas (sin estudios) y pertenecientes a clases sociales más bajas (categoría V), mostraron un menor nivel de apoyo de los compañeros ( $r h o=-0,48$ y $-0,46$ respectivamente) e influencia $(\mathrm{rho}=-0,63$ y $-0,76)$ y mayor inseguridad ( $\mathrm{rho}=0,38$ y 0,45$)$. En las ocupaciones con trabajadores más jóvenes se observa menor influencia $(\mathrm{rho}=-0,24)$, mientras que en las ocupaciones con mayor proporción de extranjeros se observa menor apoyo de los compañeros $(\mathrm{rho}=-0,44)$ y también menor influencia $(\mathrm{rho}=-0,43)$.

Por otro lado, las ocupaciones con mayor número de empleadores y autónomos y que realizan con más frecuencia horas extraordinarias, tenían un mayor nivel de influencia en el trabajo (rho=0,40, 0,33 y 0,49 respectivamente), mientras que ocupaciones con más asalariados presentaban menor nivel $\left(\mathrm{rho}_{=}=0,38\right)$. Las que tenían mayor proporción de empleo temporal presen- taban menores niveles de influencia $\left(\mathrm{rho}^{-}-0,53\right)$ y apoyo de los compañeros $(\mathrm{rho}=-0,34)$ y las que realizaban con mayor frecuencia horas extraordinarias, mayor influencia $(r h o=0,49)$. El ritmo de trabajo, sólo se ha relacionado de forma significativa, pero con correlaciones bajas $(<30)$, con la situación de empleo, siendo en general más elevado en las ocupaciones con más empleadores y con mayor número de horas semanales de trabajo.

Aunque no se ha encontrado relación entre la distribución por sexo y la exposición a los factores psicosociales, encontramos que la ocupación de "Empleadas de hogar" (CNO-94: 911), donde el 99\% de los trabajadores son mujeres, presenta uno de los niveles de apoyo de los compañeros y de influencia más bajo (?=38 y 35 , respectivamente) (gráficos 1a y $1 \mathrm{~b}$ ). También se ha encontrado un nivel bajo de influencia en el trabajo $(\bar{x}=31)$ en una ocupación mayoritariamente ocupada por trabajadores españoles (99\%), la de "Ordenanzas" (gráfico 1b), y niveles altos de inseguridad ( $\overline{\mathrm{x}}=57$ ) en otra ocupación donde la totalidad de los empleados son de estudios universitarios y de la clase social I, la de "Profesionales en organización y administración de empresas” (gráfico 1c). En el gráfico $1 \mathrm{~d}$, se observa que entre las ocupaciones con ritmo de trabajo elevado se encuentran tanto ocupaciones universitarias, de clase social I y mayoritariamente masculinas, por ejemplo "Dirección de departamento de producción”, como menos cualificadas y con mayor representación del sexo femenino, "Cocineros".

\section{DISCUSIÓN}

Según la información contenida en MatEmESp, referida a ocupaciones españolas en el año 2005, los trabajadores españoles declaran un "buen ambiente psicosocial", valorando positivamente en su puesto de trabajo el apoyo recibido de los superiores y de los compañeros, la estima, posibilidad de desarrollo; y considerando niveles no elevados de inseguridad, exigencias emocionales o exigencias cualitativas. Aunque valoran negativamente el nivel de influencia en el trabajo (autonomía en el trabajo) y el de ritmo de trabajo. En general, las ocupaciones manuales presentan un peor contexto psicosocial excepto en lo referido a influencia (autonomía en el trabajo) y ritmo de trabajo. En general, las ocupaciones manuales presentan un peor contexto psicosocial, aunque niveles altos de inseguridad y ritmo de trabajo se encontraban también en ocupaciones más cualificadas. Las ocupaciones con mayor proporción de personas trabajadoras extranjeras, jóvenes, asalariadas y temporales, también mostraron mayor exposición a riesgos psicosociales. No se ha encontrado una relación significativa entre la exposición a factores psicosociales en el trabajo y la distribución de trabajadores en las ocupaciones según sexo, aunque se han encontrado ejemplos de ocupaciones muy feminizadas con un adverso contexto psicosocial. Tampoco se encuentra correlación entre la exposición a ritmo de trabajo y ningunas de las características sociodemográficas y laborales analizadas, lo que posiblemente se deba a que algunas ocupaciones son muy heterogéneas, incluyendo a personas trabajadoras de características sociodemográficas y laborales muy dispares.

Estudios previos apoyan los resultados obtenidos. La falta de apoyo de los compañeros se ha encontrado en trabajadores no cualificados y trabajadores del transporte ${ }^{18,19}$, niveles bajos de influencia en trabajadores manuales no cualificados, trabajadores de la industria y la construcción ${ }^{18,20}$ y alta inseguridad en tra- 
bajadores no cualificados ${ }^{18}$. El ritmo elevado de trabajo también se ha encontrado en el sector de hostelería, en ocupaciones relacionadas con la dirección de empresas ${ }^{18,19}$ y en supervisores de trabajadores de la construcción ${ }^{20}$.

Por otro lado, también se ha encontrado, menor apoyo entre peones de la agricultura ${ }^{18}$, mayor ritmo de trabajo en los supervisores de la construcción ${ }^{20}$ y en telefonistas, altas demandas y baja influencia en el trabajo ${ }^{21}$. Aunque no se reflejen en las tablas, en nuestro estudio, los peones de agricultura también mostraron una puntuación en apoyo de los compañeros por debajo de la media; los albañiles, baja influencia y los encargados de construcción, puntuaciones algo superiores a la media de ritmo de trabajo e influencia en el trabajo. La ocupación de telefonista $(\mathrm{CNO}=452)$ también mostró baja influencia, pero no un ritmo de trabajo elevado, este código de ocupación agrupa otras ocupaciones como por ejemplo recepcionistas en agencia de viajes, pudiéndose haberse enmascarado el nivel de exposición en estos trabajadores.

También se ha evidenciado previamente que en clases sociales más bajas se concentran las peores condiciones psicosociales ${ }^{12,22}$, con la excepción de las demandas psicológicas, que se concentran en las clases sociales más altas ${ }^{22}$. Estudios previos también han encontrado relación entre riesgos psicosociales y edad, los más jóvenes tienen menor influencia y mayor inseguridad y los mayores menor apoyo de los compañeros ${ }^{23}$; país de origen: las empleadas de hogar inmigrantes tienen peor contexto psicosocial ${ }^{24}$; situación de empleo: asalariados menor influencia y más demandas ${ }^{18}$; trabajo temporal: altas demandas, menor control y mayor inseguridad ${ }^{25}$; jornada parcial, menor apoyo de los compañeros, influencia y mayor inseguridad ${ }^{18}$ y jornadas más largas, mayores demandas pero mayor influencia ${ }^{26}$. En nuestros análisis, no se han encontrado algunas de estas relaciones de forma significativa, pero en general, los resultados apuntan en la misma dirección.

Algunos estudios han encontrado diferencias de género en la frecuencia de exposición a riesgos psicosociales, las mujeres tienen mayor ritmo de trabajo ${ }^{27}$, menor apoyo social ${ }^{28}$, menor control en el trabajo y mayor inseguridad en el empleo ${ }^{29,30}$. La VI $\mathrm{EECT}^{18}$ tampoco encuentran diferencias de género en la inseguridad laboral. Algunos estudios han concluido que las diferencias de género en la percepción de riesgos psicosociales se deben más a la diferente distribución del sexo en las ocupaciones que al género del trabajador ${ }^{30}$, aunque Hoffman si encuentra estas diferencias en la percepción en el mismo puesto de trabajo ${ }^{31}$. En este estudio se han encontrado algunas ocupaciones muy feminizada, con un contexto psicosocial desfavorable. Aunque MatEmESp incluye información desagregada por sexo sobre condiciones de empleo y agentes ergonómicos y de seguridad, no es así para factores psicosociales, por tanto, no podemos comprobar si existen diferencias de género en la percepción de riesgos que no se expliquen por la ocupación desempeñada.

Entre las limitaciones del estudio se encuentra que la información se refiere al año 2005, podría no ser adecuado la extrapolación de los datos a la actualidad. En los últimos años, y sobre todo como consecuencia de la crisis económica, las condiciones laborales han empeorado en ciertos aspectos como una mayor inseguridad y una menor autonomía y apoyo de los compañe$\operatorname{ros}^{12}$. Se debe tener en cuenta además, que MatEmESp contiene información sobre la exposición a factores psicosociales para un número limitado de ocupaciones y que el análisis que permiten

TABLA 1

Niveles $^{a}$ de exposición a riesgos psicosociales incluidos en MatEmESp.

Agente

Definición

$\operatorname{Nivel}^{\mathrm{b}}(\overline{\mathrm{x}} ; \mathrm{DT} ; \mathrm{MD})$

Mayor valor situación más desfavorable

Ritmo de trabajo Realizar el trabajo a un ritmo elevado.

50,$8 ; 28,1 ; 50,0$

Inseguridad y empleabilidad Preocupación por el cambio de condiciones de trabajo y por la dificultad de encontrar empleo si lo pierde.

42,$0 ; 29,2 ; 50,0$

\begin{tabular}{ll}
\hline Exigencias emocionales & $\begin{array}{l}\text { Exigencias para no involucrarse en la situación emocional } \\
\text { derivada de las relaciones interpersonales que implica el trabajo. }\end{array}$
\end{tabular}

Exigencias emocionales $\quad \begin{aligned} & \text { Exigencias para no involucrarse en la situación emocional } \\ & \text { derivada de las relaciones interpersonales que implica el trabajo. }\end{aligned}$

35,$7 ; 25,6 ; 33,3$ \begin{tabular}{lll}
\hline Exigencias cuantitativas & $\begin{array}{l}\text { Relación entre la capacidad de trabajo y el tiempo disponible } \\
\text { para realizarlo. }\end{array}$ & 31,$3 ; 21,3 ; 33,3$
\end{tabular}

Mayor valor situación más favorable

Apoyo social compañeros Recibir la ayuda necesaria y cuando se necesita por parte de los compañeros/as de trabajo.

\begin{tabular}{llr}
\hline Apoyo social supervisor & Recibir la ayuda necesaria, y cuando se necesita, por parte de superiores. & 65,$0 ; 26,0 ; 75,0$ \\
\hline Estima & Respeto, reconocimiento y trato justo en el trabajo. & 66,$3 ; 19,9 ; 68,8$ \\
\hline Posibilidad de desarrollo & $\begin{array}{l}\text { Oportunidades del trabajo para poner en práctica conocimientos, } \\
\text { habilidades y experiencia de los trabajadores y adquirir nuevos. }\end{array}$ & 58,$2 ; 26,0 ; 58,3$ \\
\hline
\end{tabular}

Influencia

Margen de autonomía en el trabajo.

46,$8 ; 27,7 ; 50,0$

Fuente: MatEmESp (www.matemesp.org)

$\overline{\mathrm{x}}$ : Media. DT: Desviación típica. MD: Mediana.

b Escala estandarizada de 0-100.

${ }^{a}$ Media, desviación típica y mediana para el conjunto de la población trabajadora (todas las ocupaciones) 
los datos de esta matriz es siempre agregado, siendo la unidad de observación la ocupación (no la persona trabajadora). La agrupación de las ocupaciones basada en una clasificación de ocupaciones como la CNO-94 tiene también sus limitaciones para un análisis de este tipo ${ }^{32}$.

Los resultados de este trabajo han permitido dar a conocer aquellas ocupaciones con mayores niveles de exposición a riesgos psicosociales, así como los colectivos más afectados, principalmente trabajadores manuales, apoyando la hipótesis de que las desigualdades en salud tienen una fuerte relación con las desigualdades en la exposición ocupacional. Esta información sin duda puede llevar a una mejora en la aplicación de medidas preventivas al localizar ocupaciones y colectivos específicos con mayores riesgos.

\section{TABLA 2}

Ocupaciones con mayor nivel de exposición a factores psicosociales, MatEmESp, 2005.

\section{Ocupación $^{\mathrm{a}}$}

Nivel de exposición ${ }^{b}$

\begin{tabular}{|c|c|c|c|c|c|}
\hline CNO & Descripción & $\mathrm{AC}$ & INF & INS & RT \\
\hline 112 & Dirección de departamento de producción. & & & & 64,9 \\
\hline 140 & Gerencia de otras empresas $<10$ asalariados. & & & & 62,1 \\
\hline 239 & Otros profesionales del derecho. & & & 53,7 & \\
\hline 241 & Profesionales admón. de empresas. & & & 57,1 & \\
\hline 501 & Cocineros y otros preparadores de comidas. & & & & 64,3 \\
\hline 502 & Camareros, bármanes. & & & & 63,3 \\
\hline 503 & Jefes de cocineros, de camareros. & & & & 64,1 \\
\hline 512 & Trabajadores del cuidado de personas. & 60,1 & & & \\
\hline 729 & Otros trabajadores de acabado de construcción. & & & 56,9 & \\
\hline 836 & Operadores de máquinas textiles. & & & 54,3 & \\
\hline 837 & Operadores de máquinas de alimentos. & & & 58,4 & \\
\hline 863 & Conductores de camiones. & 61,1 & & & \\
\hline 911 & Empleados del hogar. & 38,2 & & & \\
\hline 912 & Personal de limpieza de oficinas. & 58,6 & 31,4 & & \\
\hline 921 & Conserjes de edificios, limpiacristales. & 57,9 & & & \\
\hline 932 & Ordenanzas. & & 30,8 & & \\
\hline 935 & Recogedores de basura. & & 31,7 & & \\
\hline 960 & Peones de la construcción. & & 30,6 & & \\
\hline 970 & Peones de industrias manufactureras. & & 32,0 & & \\
\hline
\end{tabular}

${ }^{a}$ Códigos de ocupación a tres digitos de la Clasificación Nacional de Ocupaciones (CNO-94).

b Se muestra las cinco ocupaciones con los valores más alejados, en sentido desfavorable, de la media de exposición (Tabla 1) a Inseguridad (INS), Ritmo de trabajo (RT), Apoyo de los compañeros (AC) e Influencia (INF), para el conjunto de ocupaciones analizadas, en España en 2005. 
TABLA 3

Correlación ${ }^{\mathrm{a}}$ entre la exposición a factores psicosociales y las características sociodemográficas y las condiciones de empleo de la población trabajadora, por ocupación, 2005.

\begin{tabular}{|c|c|c|c|c|c|c|c|c|}
\hline & \multicolumn{2}{|c|}{ Apoyo compañeros } & \multicolumn{2}{|c|}{ Influencia } & \multicolumn{2}{|c|}{ Inseguridad } & \multicolumn{2}{|c|}{ Ritmo } \\
\hline & rho & $\mathrm{p}$ & rho & $\mathrm{p}$ & rho & $\mathrm{p}$ & rho & $\mathrm{p}$ \\
\hline Mujeres & 0,07 & 0,529 & $-0,01$ & 0,954 & $-0,03$ & 0,759 & $-0,07$ & 0,514 \\
\hline \multicolumn{9}{|l|}{ Edad } \\
\hline$<31$ & $-0,04$ & 0,747 & $-0,24$ & 0,033 & 0,13 & 0,252 & 0,04 & 0,714 \\
\hline $31-45$ & 0,21 & 0,054 & 0,32 & 0,004 & $-0,08$ & 0,459 & $-0,06$ & 0,574 \\
\hline$>45$ & $-0,07$ & 0,525 & 0,1 & 0,398 & $-0,08$ & 0,482 & 0,01 & 0,929 \\
\hline \multicolumn{9}{|l|}{ Nivel de estudios } \\
\hline Sin estudios & $-0,48$ & $<0,001$ & $-0,63$ & $<0,001$ & 0,38 & 0,001 & 0,19 & 0,090 \\
\hline Primarios & $-0,43$ & $<0,001$ & $-0,56$ & $<0,001$ & 0,31 & 0,004 & 0,15 & 0,176 \\
\hline Secundarios & 0,04 & 0,691 & $-0,3$ & 0,006 & 0,1 & 0,364 & 0,08 & 0,497 \\
\hline Universitarios & 0,32 & $<0,003$ & 0,54 & $<0,001$ & $-0,27$ & 0,015 & $-0,17$ & 0,134 \\
\hline Extranjeros & $-0,44$ & $<0,001$ & $-0,43$ & $<0,001$ & 0,15 & 0,186 & 0,05 & 0,633 \\
\hline Clase Social (I-V) & $-0,46$ & $<0,001$ & $-0,73$ & $<0,001$ & 0,45 & $<0,001$ & 0,07 & 0,546 \\
\hline \multicolumn{9}{|l|}{ Situación empleo } \\
\hline Asalariados & 0,06 & 0,596 & $-0,38$ & 0,000 & 0,22 & 0,049 & $-0,27$ & 0,014 \\
\hline Autónomos & $-0,04$ & 0,738 & 0,33 & 0,003 & $-0,15$ & 0,183 & 0,10 & 0,396 \\
\hline Ayuda familiar & 0,04 & 0,692 & $-0,03$ & 0,822 & $-0,01$ & 0,898 & 0,13 & 0,249 \\
\hline Empleadores & $-0,03$ & 0,786 & 0,40 & 0,000 & $-0,09$ & 0,401 & 0,25 & 0,022 \\
\hline Contrato temporal & $-0,34$ & 0,002 & $-0,53$ & 0,000 & 0,19 & 0,086 & $-0,02$ & 0,881 \\
\hline Jornada parcial & $-0,08$ & 0,487 & $-0,11$ & 0,344 & $-0,07$ & 0,542 & $-0,16$ & 0,154 \\
\hline Horas semanales & $-0,06$ & 0,578 & 0,19 & 0,094 & $-0,01$ & 0,898 & 0,25 & 0,027 \\
\hline Horas extras & 0,06 & 0,605 & 0,49 & 0,000 & $-0,17$ & 0,121 & 0,10 & 0,382 \\
\hline
\end{tabular}

${ }^{a}$ En negrita las relaciones significativas $(\mathrm{p} \leq 0,05)$ y rho $\geq 0,30$. A menor puntuación en apoyo e influencia situación de exposición más desfavorable. rho: Coeficiente de correlación no paramétrico de Spearman.

p: P-valor. 
FIGURA 1

Relaciones entre la distribución de trabajadores por ocupación según características personales y el nivel de exposición a riesgos psicosociales en las distintas ocupaciones.

Figura 1a

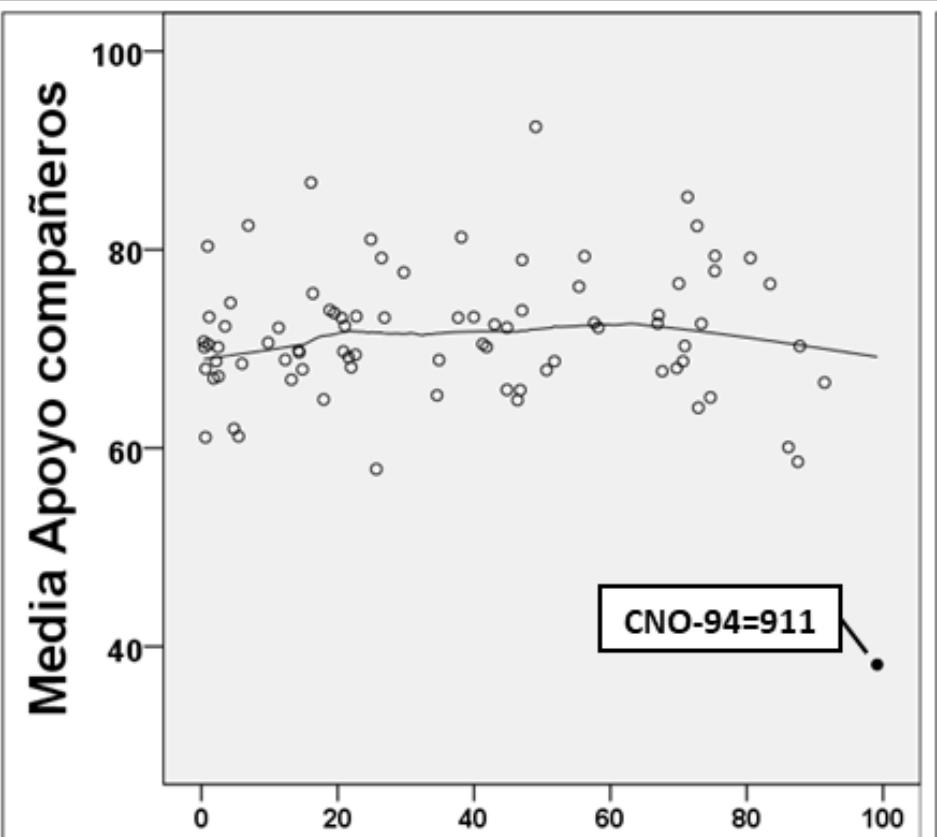

$\%$ Mujeres por ocupación

Figura 1c

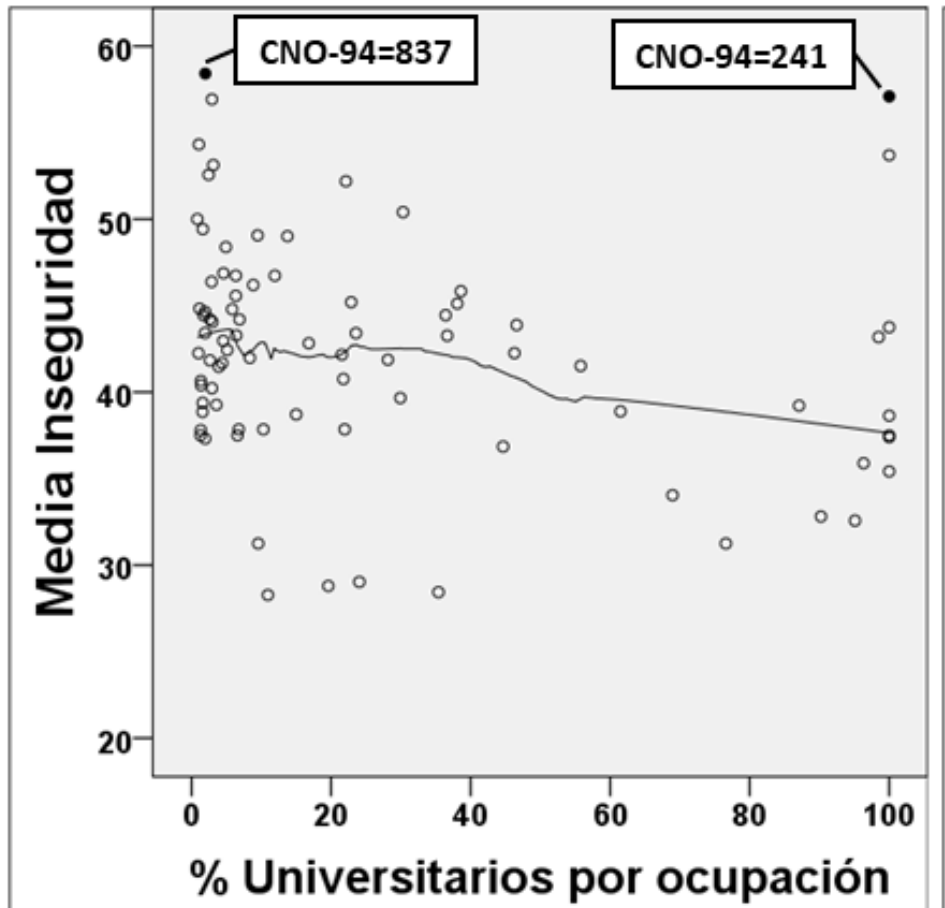

Figura 1b

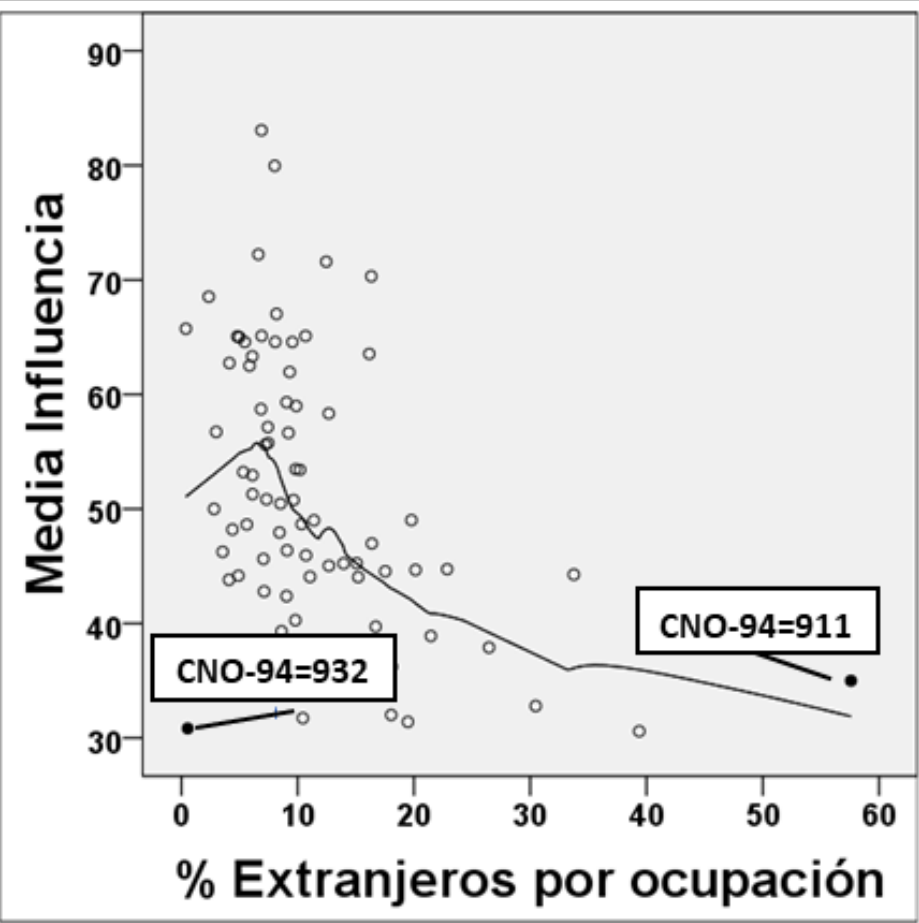

Figura 1d

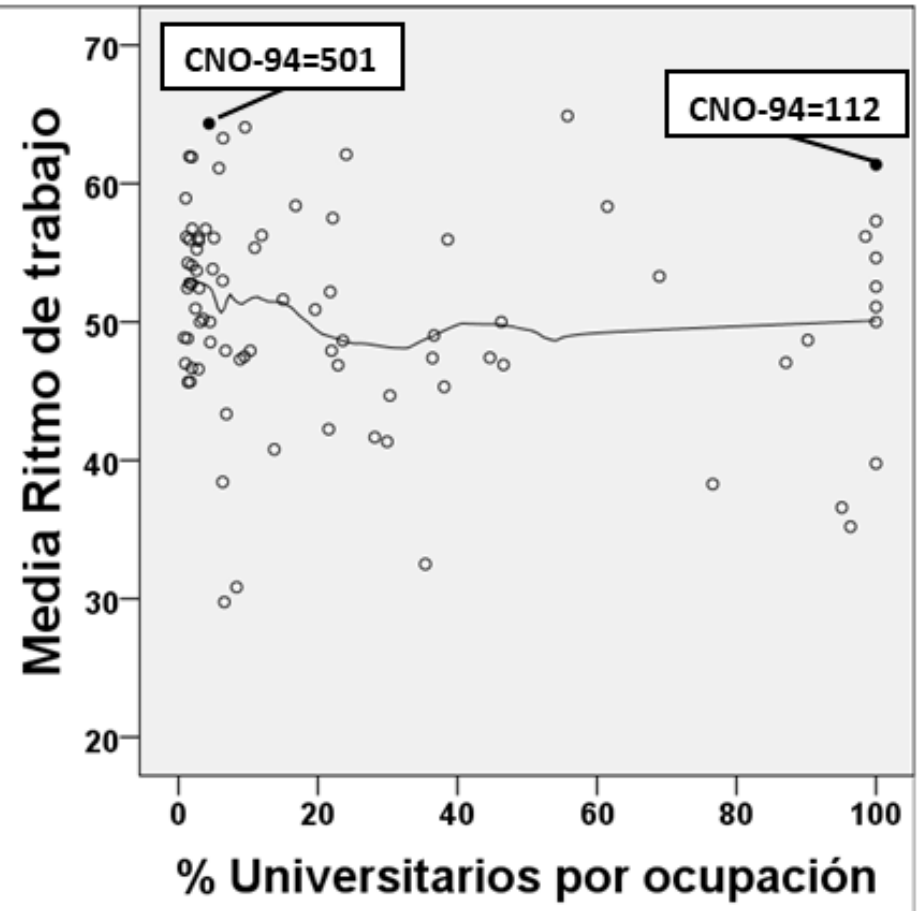

Fuente: MatEmESp (Matriz Empleo Exposición Española), www.matemesp.org.

CNO (Código Nacional de Ocupaciones) 1994=112: Dirección de departamento de producción; 241: Profesionales en organización y administración de empresas; 501: Cocineros; 837: Operadores de máquinas para elaborar productos alimenticios, bebidas y tabaco; 911: Empleadas de hogar: 932: Ordenanzas. 


\section{BIBLIOGRAFÍA}

1. Kauppinen T, Toikkanen J, Pukkala E. From cross-tabulations to multipurpose exposure information systems: a new job-exposure matrix. Am J Ind Med. 1998;33:409-17.

2. Santibañez M, Alguacil J, García de la Hera M, Navarrete-Muñoz EM, Llorca J, Aragonés N, et al. Occupational exposures and risk of stomach cancer by histological type. Occup Environ Med. 2012;69:268-75.

3. González-Galarzo M, García A, Benavides F. Características demográficas y profesionales de los usuarios de la Matriz Empleo-Exposición Española (MatEmESp). Segur Salud En El Trab. 2015;82:24-31.

4. García A, González-Galarzo M, Kauppinen T, Delclos GL, Benavides FG. A job-exposure matrix for research and surveillance of occupational health and safety in Spanish workers: MatEmESp. Am J Ind Med. 2013;56:1126-38.

5. Wieclaw J, Agerbo E, Mortensen PB, Burr H, Tuchsen F, Bonde JP. Psychosocial working conditions and the risk of depression and anxiety disorders in the Danish workforce. BMC Public Health. 2008;8:280. doi: 10.1186/1471-2458-8-280. 6. Niedhammer I, Chastang J-F, Levy D, David S, Degioanni S, Theorell T. Study of the validity of a job-exposure matrix for psychosocial work factors: results from the national French SUMER survey. Int Arch Occup Environ Health. 2008;82:87-97.

7. Johnson JV, Stewart WF. Measuring work organization exposure over the life course with a job-exposure matrix. Scand J Work Environ Health. 1993;19:21-8. 8. González-Galarzo M, García A, Gadea Merino R, Martínez Martínez JM, Velarde Collado JM. Exposición a carga física en el trabajo por ocupación: una explotación de los datos en matriz empleo-exposición española (MATEMESP). Rev Esp Salud Pública. 2013;87:601-14.

9. Lope V, García-Pérez J, Pérez-Gómez B, Pedraza-Flechas AM, Alguacil J, González-Galarzo MC, et al. Occupational exposures and mammographic density in Spanish women. Occup Environ Med. 2018;75:124-31.

10. MatEmESp: Matriz Empleo/Exposición Española [página principal de Internet]. 2012. [citado 6 marzo de 2019]. Disponible en: http://matemesp.org.

11. Torá I, Martínez JM, Benavides FG, Leveque K, Ronda E. Effect of economic recession on psychosocial working conditions by workers' nationality. Int J Occup Environ Health. 2015;21:328-32.

12. Utzet M, Moncada S, Molinero E, Llorens C, Moreno N, Navarro A. The changing patterns of psychosocial exposures at work in the South of Europe: Spain as a labor market laboratory. Am J Ind Med. 2014;57:1032-42.

13. Theorell T, Jood K, Järvholm LS, Vingård E, Perk J, Östergren PO, et al. A systematic review of studies in the contributions of the work environment to ischaemic heart disease development. Eur J Public Health. 2016;26:470-7.

14. Niedhammer I, Lesuffleur T, Algava E, Chastang J-F. Classic and emergent psychosocial work factors and mental health. Occup Med Oxf Engl. 2015;65:126-34.

15. Lang J, Ochsmann E, Kraus T, Lang JWB. Psychosocial work stressors as antecedents of musculoskeletal problems: A systematic review and meta-analysis of stability-adjusted longitudinal studies. Soc Sci Med. 2012;75:1163-74.

16. Heikkilä K, Fransson EI, Nyberg ST, Zins M, Westerlund H, Westerholm P, et al. Job strain and health-related lifestyle: findings from an individual-participant meta-analysis of 118,000 working adults. Am J Public Health. 2013;103:2090-7. 17. Domingo-Salvany A, Regidor E, Alonso J, Alvarez-Dardet C, Borrell, C, Doz, F, et al. Una propuesta de medida de la clase social. Grupo de Trabajo de la Sociedad Española de Epidemiologia y de la Sociedad Española de Medicina de Familia y Comunitaria. Aten Primaria. 2000;25:350-63.

18. Parent-Thirion A, Biletta I, Cabrita J, Vargas O, Vermeylen G, Wilczynska A, et al. Sixth European Working Conditions Survey [edición electrónica]. Luxemburgo: Eurofound; 2017 [citado 6 Mar 2019]. Disponible en: https://www. eurofound.europa.eu/sites/default/files/ef_publication/field_ef_document/ ef1634en.pdf.

19. Stauder A, Nistor K, Zakor T, Szabó A, Nistor A, Ádám S, et al. Quantifying Multiple Work-Related Psychosocial Risk Factors: Proposal for a Composite Indicator Based on the COPSOQ II. Int J Behav Med. 2017;24:915-26.

20. Boschman JS, van der Molen HF, Sluiter JK, Frings-Dresen MHW. Psychosocial work environment and mental health among construction workers. Appl Ergon. 2013;44:748-55.

21. Croidieu S, Charbotel B, Vohito M, Renaud L, Jaussaud J, Bourboul C, et al. Call-handlers' working conditions and their subjective experience of work: a transversal study. Int Arch Occup Environ Health. 2008;82:67-77.

22. Niedhammer I, Lesuffleur T, Labarthe G, Chastang J-F. Role of working conditions in the explanation of occupational inequalities in work injury: findings from the national French SUMER survey. BMC Public Health. 2018;18:344. 23. Mullan, J, Vargas, O, Willkens, M. Working conditions of workers of different ages: European Working Conditions Survey 2015 [edición electrónica]. Lu- xemburgo: Eurofound; 2017 [citado 6 Mar 2019]. Disponible en: https://www. eurofound.europa.eu/sites/default/files/ef_publication/field_ef_document/ ef1747en.pdf.

24. Ahonen EQ, López-Jacob MJ, Vázquez ML, Porthé V, Gil-González D, García AM, et al. Invisible work, unseen hazards: The health of women immigrant household service workers in Spain. Am J Ind Med. abril de 2010;53:405-16.

25. Roquelaure Y, Petit LeManach A, Ha C, Poisnel C, Bodin J, Descatha A, et al. Working in temporary employment and exposure to musculoskeletal constraints. Occup Med. 2012;62:514-8.

26. Grosch JW, Caruso CC, Rosa RR, Sauter SL. Long hours of work in the U.S.: Associations with demographic and organizational characteristics, psychosocial working conditions, and health. Am J Ind Med. 2006;49:943-52.

27. Eng A, 't Mannetje A, McLean D, Ellison-Loschmann L, Cheng S, Pearce N. Gender differences in occupational exposure patterns. Occup Environ Med. 2011;68:888-94.

28. Cohidon C, Niedhammer I, Wild P, Guéguen A, Bonenfant S, Chouanière D. Exposure to job-stress factors in a national survey in France. Scand J Work Environ Health. 2004;30:379-89.

29. Moncada S, Pejtersen JH, Navarro A, Llorens C, Burr H, Hasle P, et al. Psychosocial work environment and its association with socioeconomic status. A comparison of Spain and Denmark. Scand J Public Health. 2010;38:137-48. 30. Campos-Serna J, Ronda-Pérez E, Artazcoz L, Moen BE, Benavides FG. Gender inequalities in occupational health related to the unequal distribution of working and employment conditions: a systematic review. Int J Equity Health. 2013;12:57. doi: 10.1186/1475-9276-12-57.

31. Hooftman WE, van der Beek AJ, Bongers PM, van Mechelen W. Gender differences in self-reported physical and psychosocial exposures in jobs with both female and male workers. J Occup Environ Med. 2005;47:244-52.

32. Kromhout H, Vermeulen R. Application of job-exposure matrices in studies of general population: some clues to their performance. Eur Respir Rev. 2001;11:80-90. 